\section{The European Spallation Source}

\author{
H. Lengeler, CERN and KFA, Jülich (ESS Project Leader) \\ J.L. Finney, University College, London (ESS Science Co-ordinator)
}

\begin{abstract}
As we move towards the 21st Century, the complexity of technology and of the scientific questions posed will increasingly require detailed knowledge at the atomic level. Neutrons are one of the key microscopic techniques with which an improved understanding of physics and materials can be gained, and to fully capitalise on this potential, higher intensity sources are crucial.
\end{abstract}

The neutron is a powerful and versatile probe of both the structure and dynamics of condensed matter. Together with other established techniques such as X-ray, electron, and light scattering, and emergent techniques such as scanning-probe microscopy, the neutron has provided basic microscopic information which underpins our understanding of not only the physics of condensed matter, but also condensed matter in fields as diverse as biology, materials science, chemistry, and the earth sciences. Although often exploited as a technique complementary to X-ray scattering, the interaction of the neutron with matter is unique, and therefore capable of providing information which may be either difficult or impossible to obtain in other ways (see insert).

But neutrons have one very serious disadvantage: neutron scattering is a seriously intensity-limited technique. The power and versatility of the neutron is, however, such that a significant enhancement of source brightness would allow neutrons to be used to answer questions that presently cannot be tackled in areas involving high-density magneto-optical storage devices, chemistry related to pollution, the behaviour of small molecules that protect enzymes from denaturation under conditions of stress, and stronger, lighter, and more reliable engineering components.

Looking back historically, we can see that increases in source intensity have invariably given rise to major expansion of the science that can usefully be tackled by a given technique. In the neutron case, the technique really began to blossom from the mid-1970s with the coming on-stream of the high-flux reactor at the Institut Laue-Langevin (ILL) in Grenoble. The source intensity was such that a major expansion in the use of neutrons took place, putting European neutron science into a world-leading position.

Neutron techniques being inherently intensity limited, there is therefore a strong scientific incentive towards more intense sources. Unfortunately, reactor production of neutrons seems to be coming up against the major engineering limitation of heat extraction from the reactor core. Studies in the USA over recent years have resulted in a design for an enhanced reactor source - the Advanced Neutron Source (ANS) - which will deliver an effective neutron flux of up to five times that of the ILL. This does, however, seem to be the end of the line for reactor sources.

\section{Accelerator Sources}

An alternative line of development has led to the construction and use of neutron sources based on particle accelerators. In such sources at Argonne (IPNS), Los Alamos (LANSCE), KEK, Japan, and the Rutherford
Appleton Laboratory (ISIS), sharp pulses of ral hundred $\mathrm{MeV}$, and then made to impinge on a heavy metal target, where sharp pulses of neutrons are produced. Although the timeaveraged neutron flux from the most powerful such source (ISIS) is significantly lower than that of the ILL over the pulse duration, the source brightness can be significantly higher than from any of today's reactor neutron sources. Thus, in those experiments where this high temporal brightness can be exploited, these sources can further widen the problems that neutrons can tackle effectively.

We do not go here into details of the very considerable advantages of the time structure, which among others, allow in general higher resolution, structural and dynamic studies over wide dynamic ranges in space and time, high-energy excitation work, and low backgrounds. [See, for example, J.L. Finney, Europhys. News 20 (1989) 119; C.J. Carlile \& J.L. Finney, Physica B 174 (1991) 451]. protons are accelerated to energies of seve-
Fortunately, pulsed spallation sources are at an early stage of development, and there seems to be no major difficulty in building a source many times more powerful than ISIS. As a result of an initiative from the European Commission, a study for a next generation European Spallation Source (the ESS) the Rutherford Appleton Laboratory. Several meetings were held to explore options for the accelerator that would drive such a source, the target station that would produce the neutrons, and the new science that could become possible. The reference design parameter was set at a proton power of $5 \mathrm{MW}$ which, being some 30 times the $160 \mathrm{~kW}$ of ISIS, would result in a very major increase in source intensity. At this level, the proposed source would yield about the same timeaveraged neutron flux as the ILL: exploiting the advantages of the time structure, the ESS would be capable of out-performing any other source in very nearly all areas of neutron scattering.

\section{Significant Advantages}

The increase in source brightness will significantly improve the quality and quantity of information that can be obtained from neutron scattering. With more intensity, experiments can be performed on smaller samples in shorter times. This is not just to say that samples "smaller" in the physical sense can be studied (although this is an important aspect with new exotic materials, with systems subjected to extremes of temperature, pressure and external field, and with systems was initiated in 1991 by KFA Jülich and

\section{NEUTRON SCATTERING: The Main Characteristics}

Neutrons interact principally with the nucleus. Thus, as the useful neutron wavelengths are much longer than nuclear dimensions, the nucleus acts as a point scatterer. Data can therefore be taken to higher momentum transfers $Q$, allowing (crystalline and noncrystalline) structures to be studied to very high resolution. This high-resolution capability is particularly important in studying the complex structures that are increasingly common in modern materials sciences: it is no accident that neutrons have made the major contribution to understanding structures of high- $T_{\mathrm{C}}$ superconductors. Light atoms may also be studied in the presence of heavy ones - a difficult problem for X-rays. As the neutron interacts with the nucleus, significant differences may exist between the scattering powers of isotopes of the same element. Of particular interest is the large scattering length difference between $\mathrm{H}$ and $\mathrm{D}$, which can be exploited to very great effect through "contrast variation" methods to highlight particular parts of complex molecular systems. In soft-matter science in particular, neutron studies have grown explosively over the past decade, and the problems being addressed now are of major significance in the industrial world.

The energy and momentum of neutrons may be matched simultaneously to energy and length scales appropriate for excitations in condensed matter. Neutron wavelengths of $1-10 \AA$ are ideal for the study of interatomic correlations and the available neutron energies of 1 to $1000 \mathrm{MeV}$ allow the measurement of excitations over nine decades from neV to $\mathrm{eV}$.

The neutron's magnetic moment couples to spatial variations of the magnetization of materials on the atomic scale. Neutrons are thus ideally suited for the study of magnetic structures (and hence microscopic magnetism) and short-wavelength magnetic fluctuations. A recent example of this kind of measurement is the mapping out of the dynamic susceptibility of high- $T_{C}$ superconductors, thus directly addressing the question of electronelectron correlations in this important class of new materials.

The neutron is a weak probe, and does not significantly perturb the system. The resultant data may be compared directly with theory. In many cases, absolute cross-sections may be obtained, contrasting with the difficulties in interpreting information from strongly interacting probes such as photons or electrons.

The low absorption of neutrons and the lack of charge allows neutrons to probe the bulk of materials. This feature, which has made possible the construction of sophisticated sample environment equipment, is now being exploited in the study of residual stress in engineering components and composite materials. 
that are inherently small such as a thin film or the stress at a crack tip). Higher intensities will also allow more dilute systems to be investigated. Parametric studies (in composition, temperature, pressure, humidity, external fields, and combinations of these) will be possible in hours for measurements which today would take days or weeks and are therefore not presently feasible.

Higher sensitivity and better signal-tonoise will generalise the isotopic substitution technique and allow new weak cross-section phenomena to be investigated. Pulsed techniques will be employed to provide extremes of sample environment (pressure; magnetic and electric fields). Time-dependent relaxation phenomena and kinetic processes will be investigated effectively.

The scientific case includes many other examples where a source such as ESS is needed. Our understanding of magnetism and superconductivity would be considerably enhanced by the ability to:

- determine precise magnetic moments and detect weak incommensurate signals;

- measure simultaneously the average and local nuclear and magnetic structure of a given material;

- utilize the increased flux to probe surface and thin-layer magnetic structure;

- investigate time-dependent phenomena;

- perform magnetic and structural studies in high applied fields and/or high pressure.

In non-crystalline materials, not only will unprecedented real-space resolution be accessible, but also derivates of the pair-correlation function with respect to external variables. Moreover, the long-anticipated field of neutron Brillouin scattering will be realisable, allowing the probing of dynamics of liquids, glasses, and polymeric systems in the nonhydrodynamic regime. Qualitative improvements will be possible in our understanding of some of the fundamental problems in physics such as the evolution of magnetic behaviour from a Mott-Hubbard insulator. In two-dimensional magnetic excitations, the brightness of ESS represents a step forward which is comparable to that of going from a rotating anode $\mathrm{X}$-ray source to a synchrotron for X-ray structural studies. ESS would also open up the unexplored fields of the dynamics and kinetics of surfaces and thin films. The improvements in resolution made possible by ESS will put neutron spectroscopy on a par with IR and Raman scattering, but with the advantages of the lack of selection rules, and of being able to relate intensities to atomic displacements in a simple way. This could enable the study of the dynamics of industrial catalysis processes in real industrial environments rather than in idealized laboratory conditions.

\section{ESS Design Study}

The EC has funded a two-year European ESS design study. At present it involves participants from six countries in preparing a conceptual design, performing a site-independent costing, and identifying the $R \& D$ required to realise such a facility. The basic reference design is a source with a mean power of $5 \mathrm{MW}$, distributed over two targets with nominal pulse repetition rates of 10 and $50 \mathrm{~Hz}$, and a proton pulse length of about $1 \mu \mathrm{s}$. For a facility with an expected turnover of over 1000 experiments per year, special emphasis must be put on high availability and safety.

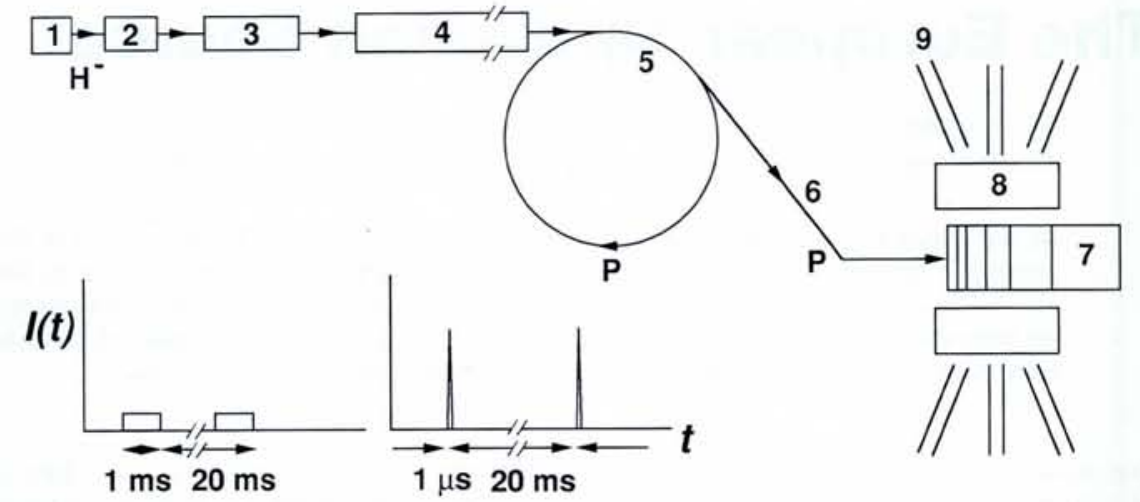

Pulsed neutron source: schematic representation showing the $H^{-}$-ion source (1), RFQ (2), drifttube linac (3), high-energy linac (4), accumulating rings with $\mathrm{H}^{*}$-charge exchange injection (5), beam transport to target (6), target (7), moderators (8), and neutron channels (9). The time structures of the beam intensty in the linear accelerator and at the target are shown in the lower part.

The basic concept of the proposed source is shown in Fig. 1. $\mathrm{H}^{-}$-ions are accelerated in a linear accelerator to approximately $1 \mathrm{GeV}$ with pulses of about $1 \mathrm{~ms}$ duration. In order to produce in the target proton pulses of length about $1 \mu \mathrm{s}$, the $\mathrm{H}^{-}$-ions are injected by charge exchange $\left(\mathrm{H}^{-} \rightarrow \mathrm{H}^{+}\right)$over many turns into an accumulator or accelerator ring. Once the required current is reached, the protons are extracted during a single turn and are then transported to the target. In this way, pulses are produced whose peak current is between two and three orders of magnitude above the steady current produced in the linear accelerator.

The accelerators have to be designed for an average beam power of several MW. This requires a considerable increase in performance over existing machines. The most powerful high-energy accelerator for protons - LAMPF at Los Alamos - accelerates an average current of up to $1 \mathrm{~mA}\left(\mathrm{H}^{+}\right)$and 100 $\mu \mathrm{A}\left(\mathrm{H}^{-}\right)$to $800 \mathrm{MeV}$, corresponding to an average power of about $800 \mathrm{~kW}$. The most powerful existing ring accelerator for protons in the GeV range is the ISIS rapid-cycling synchrotron, with a proton energy of 800 $\mathrm{MeV}$, and an average beam power of 160 $\mathrm{kW}$. For the ESS, more than one ring may be needed, depending on the final proton energy which still has to be fixed somewhere in the range between 0.8 and $3 \mathrm{GeV}$.

Developments will be necessary for both kinds of accelerator, including the construction of some prototype elements. For the linac high-intensity $\mathrm{H}^{-}$-sources, beam chopping and tunneling will have to be studied. The use of superconducting cavities is also proposed.

For ring accelerators, the problems of $\mathrm{H}^{-}$ injection and $\mathrm{H}^{+}$extraction at high beam currents need to be considered, together with the avoidance of instabilities. Special attention will have to be given to beam losses, for in a proton machine, these lead to activation of components and, if not reduced to a minimum, can render maintenance and repair difficult and costly. Relative beam losses in the ESS accelerators must be reduced to values that are lower than those that are presently achievable. Well-matched beam-transport systems will be essential in all parts of the accelerator complex, and unavoidable beam losses (e.g. at the injection regions of the ring) will be collected at well-defined and easily-accessible positions.

\section{Target Station}

The principle of the target layout is also shown in the figure. The proton beam hits a target that could be water-cooled heavy metal or circulating molten metal. In one spallation event some 10-20 neutrons per proton are produced in the energy range of a few $\mathrm{MeV}$. The neutrons leave the target and are slowed down to thermal or sub thermal energies by moderators, although to retain the important time structure, moderation is not allowed to go to completion. Each target station may serve 15 to 20 neutron channels, each of which will be host to at least one instrument.

As in the case of the accelerator, considerable development work is needed on the target station especially with respect to radiation damage and the cooling of the moderators. An additional problem is raised by the enormous proton peak currents. The energy content of the proton pulses will be of the order of $100 \mathrm{~kW}$, which may lead to large thermal and mechanical shocks in the beam windows and the target. There is little experience to hand in this matter and experimental studies will probably be necessary to help solve these problems.

\section{CERN ACCELERATOR COURSE Scholarships Offered}

In order to broaden its activities and to promote the education of young physicists and engineers in the field of accelerators, the EPS Interdivisional Group for Accelerators has decided to establish scholarships for participation in the CERN Accelerator School (CAS) Basic General Course held every two years. The scholarships should enable candidates to participate free of charge (except for travel) starting in 1994 (the next course will be held in Baden, Germany, on 19-30 September 1994). Candidates should be graduate students, young physicists or engineers from Europe who do not have a permanent position. They will be selected by the CAS Programme Committee of the General Accelerator Course.

Please apply to the CERN Accelerator School, CERN, CH-1211 Geneva 23. 